\title{
Nanodiamond decorated liposomes as highly biocompatible delivery vehicles and a comparison with carbon nanotube and graphene oxide
}

\author{
Feng Wang and Juewen Liu* \\ Department of Chemistry, Waterloo Institute for Nanotechnology, University of Waterloo, Waterloo, \\ Ontario, Canada N2L 3G1. \\ Email: liujw@uwaterloo.ca
}

\begin{abstract}
Studying interactions between nano-carbons and lipid membranes is important for multiplexed drug delivery, device fabrication and for understanding toxicity. Herein, we report that nanodiamond (ND, $s p^{3}$ carbon) forms a complex with highly biocompatible zwitterionic liposomes based on hydrogen bonding, which is confirmed by $\mathrm{pH}$-dependent and urea-dependent assays. Despite such weak interaction, the complex is highly stable. Comparisons were made with two $s p^{2}$ carbons: nanoscale graphene oxide (NGO) and carbon nanotube (CNT), where CNT adsorption is the weakest. Adsorption of the nano-carbons does not induce liposome leakage or affect lipid phase transition temperature. Therefore, the potential toxicity of nano-carbons is unlikely to be related to direct membrane damage. ND facilitates cellular uptake of liposomes and co-delivery of negatively charged calcein and positively charged doxorubicin has been demonstrated. ND has the lowest toxicity, while CNT and NGO are slightly more toxic. The effect of introducing fusogenic lipids and cholesterol was further studied to understand the effect of lipid formulation.
\end{abstract}




\section{INTRODUCTION}

In the past few decades, carbon-based nanomaterials have tremendously fueled the growth of nanotechnology with the discovery of various forms of this element. From the chemical and geometric standpoint, the recent interests are focused on the following three materials: carbon nanotubes (CNTs, 1-D, $s p^{2}$ carbon), graphene (2-D, $s p^{2}$ carbon), and nano-diamond (ND, 0-D, $s p^{3}$ carbon). These nanocarbons have been explored for biomedical applications, including imaging, drug delivery, and therapeutics. CNTs were historically the first explored, encouraged by its light absorption for hyperthermia treatment, near IR fluorescence, and drug carrying ability. ${ }^{1-4}$ Pristine graphene is very hydrophobic and cannot be dispersed as monolayered sheets in water. For biomedical applications, nanoscale graphene oxide (NGO) with oxygenated groups is often used. ${ }^{5-13}$ Recently, ND has been shown to be a highly biocompatible drug carrier. ${ }^{14-23}$ Being a $s p^{3}$ carbon based material, the surface property of ND is quite different from its $s p^{2}$ counterparts. While remarkable progresses have been made on the application side, fundamental interactions at the bio/nano interface are still not fully understood. ${ }^{24-26}$

One of the most important and fundamental aspects is their interaction with lipid membranes. Endocytosis, pore formation and oxidative stress are all related to membrane processes. Understanding membrane/nanoparticle interaction may also have direct applications in sensing, ${ }^{27}$ drug delivery, ${ }^{28}$ imaging, ${ }^{29}$ and device fabrication. Liposomes are an ideal model for the cell membrane. To attach nano-carbons to liposomes, the majority of the previous work employed electrostatic interactions or covalent linkages. For example, cationic liposomes were used to adsorb negatively charged GO. ${ }^{30}$ Liposomes of various charges were adsorbed by graphene deposited on a wafer for device fabrication. ${ }^{31}$ Molecular dynamics simulation showed the insertion of a graphene sheet between the hydrophobic tails of a bilayer, ${ }^{32}$ and the level of graphene oxidation appears to be important. ${ }^{33}$ There are also reports on interfacing liposomes with CNTs, where covalent attachment is often used. ${ }^{34-36}$ Recently, attempts were 
made to encapsulate ND inside liposomes. ${ }^{37,38}$ However, little is known about the interaction between ND and liposomes. A systematic comparison between the different nano-carbons is also lacking.

We are particularly interested in exploring non-covalent and non-electrostatic interactions, since covalent conjugation is often expensive and difficult to carry out while cationic components are always toxic. Being one of the major components in the cell membrane and highly biocompatible, zwitterionic phosphatidylcholine (PC) liposomes are particularly attractive as a model system (see Figure S1 for the lipid structures). We herein report that ND can be attached to PC liposomes by a simple mixing step via hydrogen bonding. Comparisons were made to CNT and NGO to allow mechanistic insights (Figure 1A). Using this ND/liposome complex for co-delivery of an imaging dye and an anti-cancer drug has been demonstrated.

\section{RESULTS AND DISCUSSION}

2.1. Non-electrostatic adsorption of NDs. Our ND samples were washed extensively with concentrated acids to generate surface carboxyl groups, leading to a negatively charged surface (zetapotential $=-30.5 \mathrm{mV}$ at $\mathrm{pH}$ 6.0). IR spectroscopy indicates the presence of surface carboxyl, hydroxyl, and other oxygenated species (Figure S2). ${ }^{39}$ The resulting product can be readily dispersed in water with good colloidal stability. The size of the ND particles has a relatively large distribution as indicated by TEM (Figure S3). The UV-vis spectrum of our $30 \mathrm{~nm}$ ND sample is shown in Figure 1B (red trace), where only the light scattering feature is observed and shorter wavelengths scatter more strongly. For comparison, the UV-vis spectra of NGO and CNT are also presented; both have a well-defined absorption peak in the UV region. The lack of UV absorbance of our ND sample confirms the different chemistry between $s p^{2}$ and $s p^{3}$ carbons in our samples.

To interface ND with liposomes, most previous work aimed to encapsulate ND inside liposomes via co-extrusion or sonication, ${ }^{37,38}$ where improved biocompatibility and colloidal stability of ND might be achieved. In this work, we employed a different design by decorating ND on the 
surface of liposomes. We reason that such a design leaves the internal compartment of liposomes free for drug loading while the ND surface could still adsorb drugs for multiplexed delivery, which might be a more efficient way to utilize the surface area of nanomaterials. The simplest way to attach ND is through adsorption. To test feasibility, we respectively mixed ND with cationic 1,2-dioleoyl-3trimethyl ammonium-propane (DOTAP), zwitterionic 1,2-dioleoyl-sn-glycero-3-phosphocholine (DOPC), and anionic 1,2-dioleoyl-sn-glycero-3-phospho-(1'-rac-glycerol) (sodium salt) (DOPG) liposomes, all labeled with $1 \%$ rhodamine $(\mathrm{Rh})$. After centrifugation, the supernatant contains only the free liposomes while liposomes decorated with ND are precipitated (Figure 2A). Both DOTAP and DOPC liposomes can adsorb ND since their supernatants are dark. The lack of adsorption by DOPG liposomes is explained by electrostatic repulsion. For drug delivery, DOPC is preferred over DOTAP since cationic DOTAP is highly toxic. The kinetics of ND adsorption by DOPC liposomes is quite fast and is finished in $\sim 1$ min after mixing (Figure $\mathrm{S} 4$ ). 


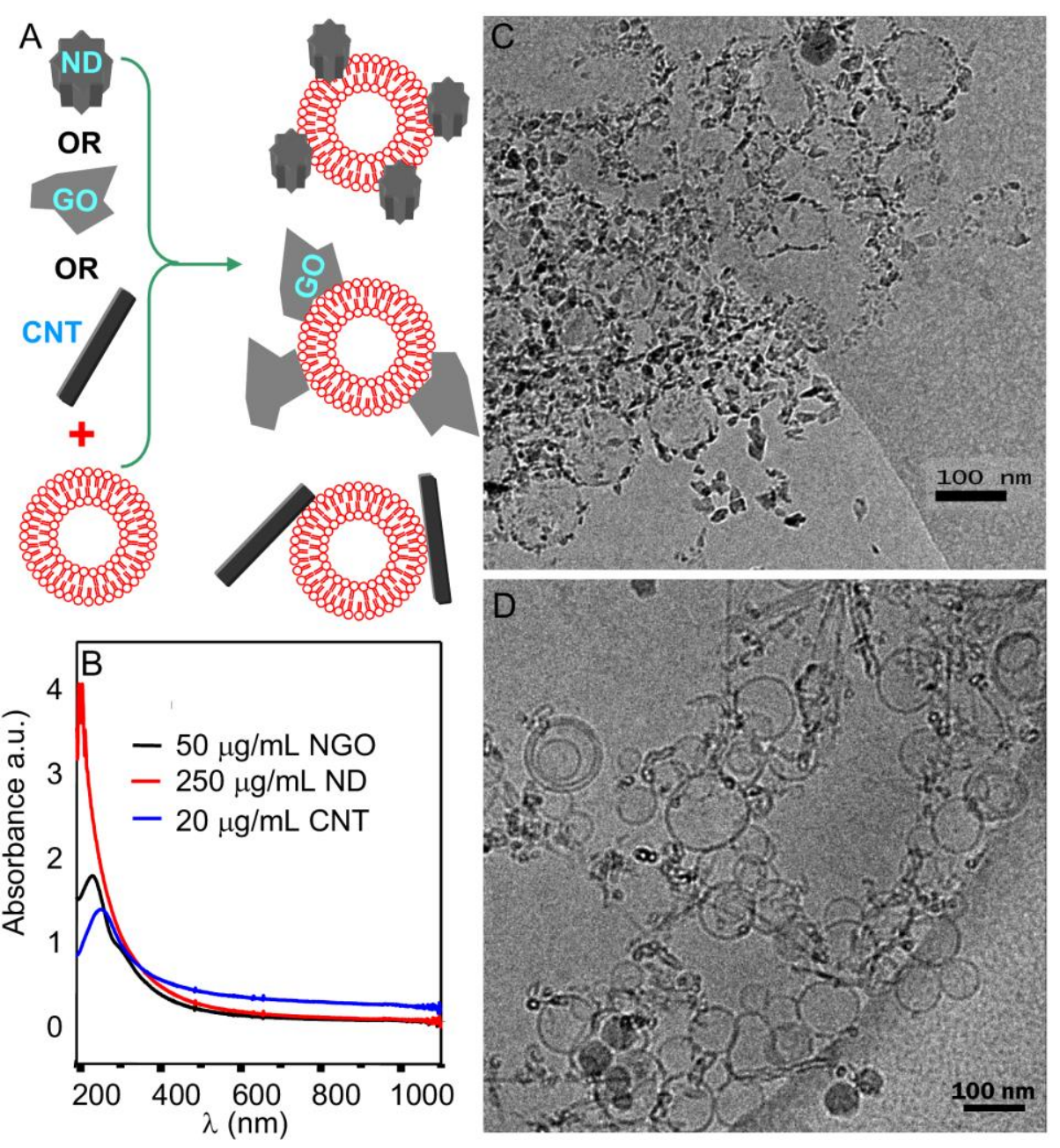

Figure 1. (A) Schematics of adsorption of the nano-carbons onto zwitterionic PC liposomes. (B) UVvis spectra of the nano-carbons. Cryo-TEM micrographs of ND/DOPC (C) and CNT/DOPC (D) hybrids. Scale bars $=100 \mathrm{~nm}$.

Next we compared the adsorption of the three nano-carbons by DOPC liposomes (Figure 2B). Based on the lack of supernatant fluorescence, all of them can adsorb DOPC liposomes. To further characterize these complexes, cryo-TEM was used. The ND particles follow the contour of the liposome surface (Figure 1C), confirming specific adsorption interaction. CNTs (single-walled) also show adsorption by DOPC liposomes and no free CNTs are observed (Figure 1D). The spherical shape of liposomes is maintained in both systems. More cryo-TEM micrographs are presented in Figure S5. 
For many applications, especially for drug delivery, particle size and colloidal stability are very important. The cryo-TEM micrographs show aggregated liposome/nano-carbons, which might be related to sample preparation and drying. To monitor nanoparticle size in solution, dynamic light scattering (DLS) was carried out (Figure 2C). The hydrodynamic size of ND is $~ 40 \mathrm{~nm}$, while NGO and CNT are slightly above $100 \mathrm{~nm}$. The size of DOPC liposomes is $\sim 100 \mathrm{~nm}$, consistent with the cryo-TEM measurement. Next we respectively added increasing concentrations of each nano-carbon to the DOPC liposomes and the size of the complex never exceeded $200 \mathrm{~nm}$ for all the samples (Figure 2D), suggesting the lack of extensive aggregation in solution. It is intriguing to observe that the average size of the DOPC/ND mixture actually decreased by adding more ND, which might be due to ND having a much stronger light scattering ability and high refractive index, dominating the light scattering signal. This hypothesis is confirmed by mixing DOPG liposomes and ND, where no interaction is expected and the size still decreases. On the other hand, when cationic DOTAP liposomes and ND are mixed, significant growth in size indicating aggregation is observed. This serves as a positive control to confirm the lack of extensive aggregation when DOPC liposomes are mixed with the nano-carbons. The ability to form a stable DOPC/ND conjugate is useful for cellular uptake of this complex (vide infra). 
A
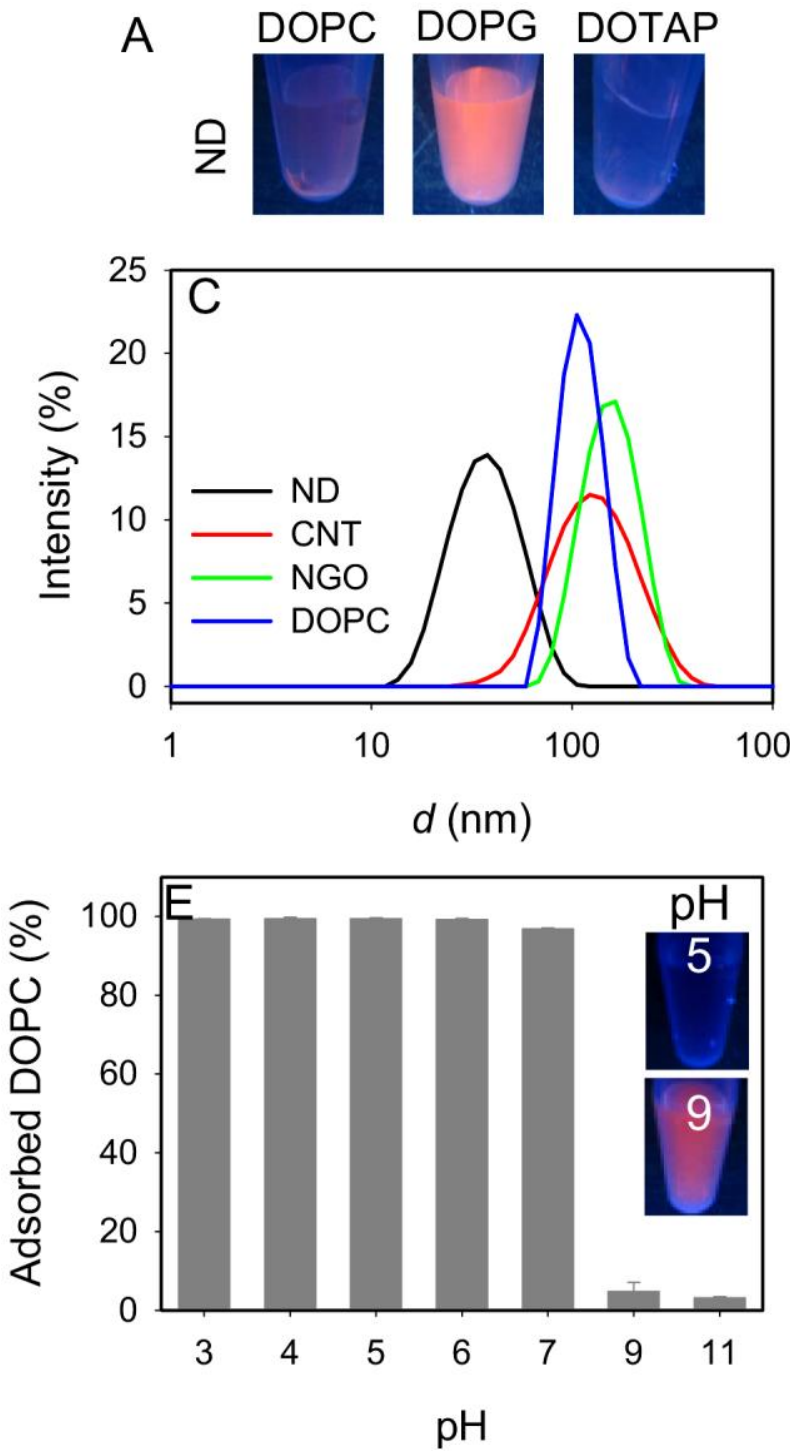

B
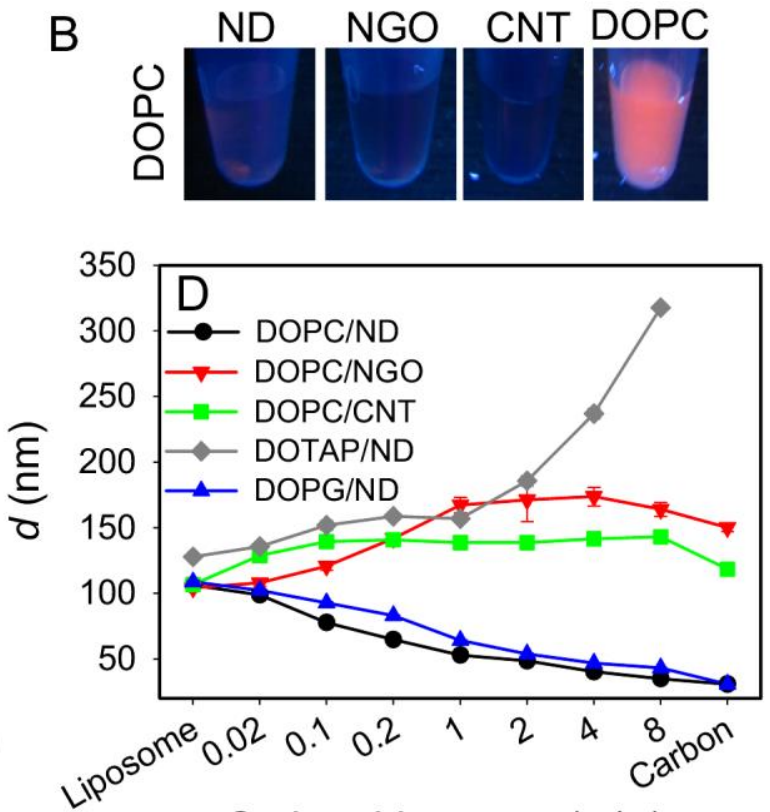

Carbon:Liposome (w/w)

$\mathrm{F}$

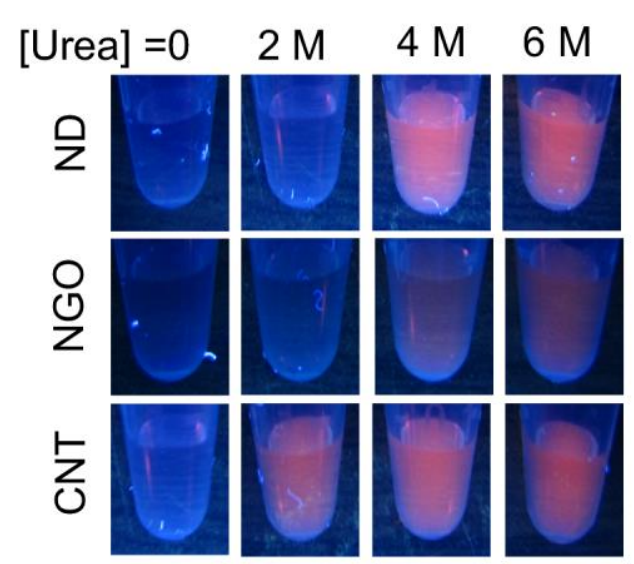

Figure 2. Photographs of ND mixed with the three types of Rh-labeled liposomes (A) and Rh-DOPC liposomes mixed with the three nano-carbons after centrifugation (B). Red fluorescence in the supernatant indicates non-adsorbed free liposomes. (C) DLS spectra of the nano-carbons and DOPC liposomes. (D) Change of the hydrodynamic size of DOPC liposomes as a function of nano-carbon concentration. (E) Adsorbed DOPC liposomes by ND as a function of $\mathrm{pH}$. Inset: sample fluorescence at pH 5 and 9. (F) Adsorption of DOPC liposomes by nano-carbons as a function of urea concentration.

2.2. Hydrogen bonding mediated adsorption. After confirming the adsorption between ND and DOPC liposomes, we aim to identify the origin of the attractive force. Electrostatic attraction or 
hydrophobic interaction is unlikely to work with zwitterionic DOPC liposomes. We propose that hydrogen bonding might be the main force for adsorption. To test it, we measured ND adsorption as a function of $\mathrm{pH}$, where stronger fluorescence from Rh-labeled DOPC liposomes or poor ND adsorption was observed at $\mathrm{pH}$ above 9 (Figure 2E). Effective adsorption was achieved at pH 7 or lower. The only possible $\mathrm{pH}$-sensitive group at around neutral $\mathrm{pH}$ is the carboxyl or other oxygenated groups on ND. To further confirm hydrogen bonding, DOPC liposomes were incubated with the nano-carbons in the presence of 0-6 M urea (Figure 2F). Interestingly, CNT desorbed with just $2 \mathrm{M}$ urea, while NGO showed slight desorption only with $6 \mathrm{M}$ urea. ND is stable in $2 \mathrm{M}$ urea but desorbs with $4 \mathrm{M}$ urea. The disruptive effect of urea further confirms the hydrogen bonding mechanism. It is likely that the protonated carboxyl is the hydrogen bond donor and the phosphate on the DOPC head group is the acceptor. The adsorption strength of DOPC liposomes follows the order of NGO > ND > CNT. Since hydrogen bond is a weak force, to realize effective adsorption, a large contact area with the liposome is required to achieve multivalent binding. The weaker adsorption of CNT might be related to its geometry and the surface carboxyl groups are unlikely to concentrate on the narrow nanotube surface to interact with the liposomes. 

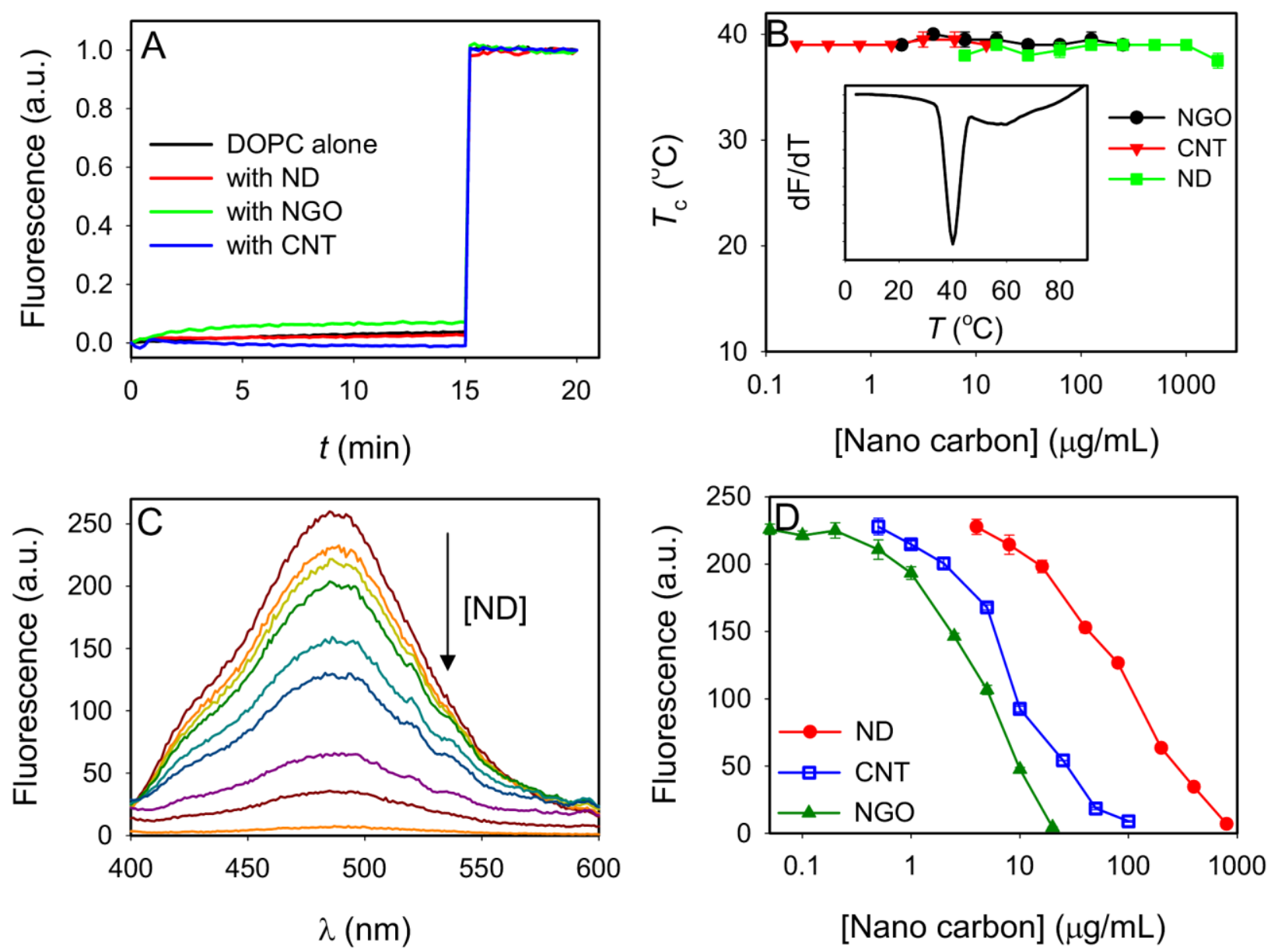

Figure 3. (A) Calcein-loaded DOPC liposome leakage test. At time zero, DOPC liposomes were mixed with various nano-carbons and at 15 min, Triton X-100 was added. (B) DPPC $T_{\mathrm{c}}$ change as a function of nano-carbon concentration. Inset: a calcein release curve as a function of temperature. (C) Laurdan fluorescence as a function of ND concentration. (D) Laurdan fluorescence intensity as a function of nano-carbon concentration.

2.3. Membrane interactions. After understanding the adsorption force, we next studied the biophysical effect of nano-carbon on the membrane, which is important for understanding toxicity. The cryo-TEM micrograph (Figure 1C) indicates that many ND particles are closely associated with the lipid membrane and important questions are whether ND induces liposome leakage and affects lipid phase transition temperature $\left(T_{\mathrm{c}}\right)$. To answer these questions, we encapsulated $100 \mathrm{mM}$ calcein inside 
DOPC liposomes to achieve the self-quenching effect. After removing free calcein, liposome leakage was monitored by fluorescence enhancement. Within $15 \mathrm{~min}$, little leakage was observed for all the test nano-carbons (Figure 3A) and full rupture was induced at $15 \mathrm{~min}$ by adding Triton X-100. To understand the effect on $T_{\mathrm{c}}$, we mixed calcein loaded 1,2-dipalmitoyl-sn-glycero-3-phospho choline (DPPC) liposomes with various concentrations of the nano-carbons. The samples were gradually heated and the fluorescence intensity was measured at each temperature. A typical response curve in the absence of nano-carbon is shown in the inset of Figure 3B, where the fastest leakage occurs at 40 ${ }^{\circ} \mathrm{C}$, consistent with the $T_{\mathrm{c}}$ of DPPC being $41{ }^{\circ} \mathrm{C}$. With increasing carbon concentration, the $T_{\mathrm{c}}$ remains constant under all the tested conditions. Therefore, these materials are likely to be adsorbed only on the liposome surface without affecting the packing of the bilayer. To further confirm this, 6-dodecanoyl-2dimethylaminonaphthalene (Laurdan) dye was embedded in the bilayer region of DOPC liposomes and its fluorescence spectra were monitored as a function of nano-carbon concentration. The Laurdan fluorescence peak was at $481 \mathrm{~nm}$, consistent with the dye sitting in a fluid bilayer. ${ }^{40-42}$ We only observed fluorescence quenching by adding nano-carbon (Figure 3C for ND; Figure S7 for NGO and CNT) without peak shifting. This also supports surface adsorption without disrupting the lipid packing. For comparison, when the dye was embedded in DPPC liposomes, the peak was at $445 \mathrm{~nm}$ at $25{ }^{\circ} \mathrm{C}$ (gel phase) and $481 \mathrm{~nm}$ at $41{ }^{\circ} \mathrm{C}$ (fluid phase) (Figure S6). Among the three nano-carbons, NGO has the strongest quenching ability, followed by CNT, while ND is a poor quencher (Figure 3D).

2.4. Multiplexed delivery. Decorating ND on DOPC liposomes offers a useful means for multiplexed delivery since both the liposomes and ND are available for drug containment. Next, we tested the cellular uptake of this hybrid. The free Rh-DOPC liposomes cannot be internalized by HeLa cells and no red fluorescence was observed (Figure 4A), while NDs of 30-100 nm could carry DOPC liposomes into the cells (Figure C, D and Figure S8). For comparison, CNT is a poor carrier for DOPC liposomes 
since only very weak red fluorescence was observed under the same imaging condition (Figure 4B). In previous reports, CNTs were linked to liposomes via biotin/avidin interaction or amide bonding to achieve co-delivery. ${ }^{34,35}$ In our case, only physisorption was employed. In particular, CNT has been shown to be only weakly adsorbed compared to ND based on the urea assay. The zero-dimension of ND might also contribute to its favorable cellular uptake compared to the 1-D CNT. Using low temperature incubation and lysosome staining dyes, we further confirmed that cellular uptake of DOPC/ND is through the energy-dependent endocytosis pathway (Figure S9, S10).
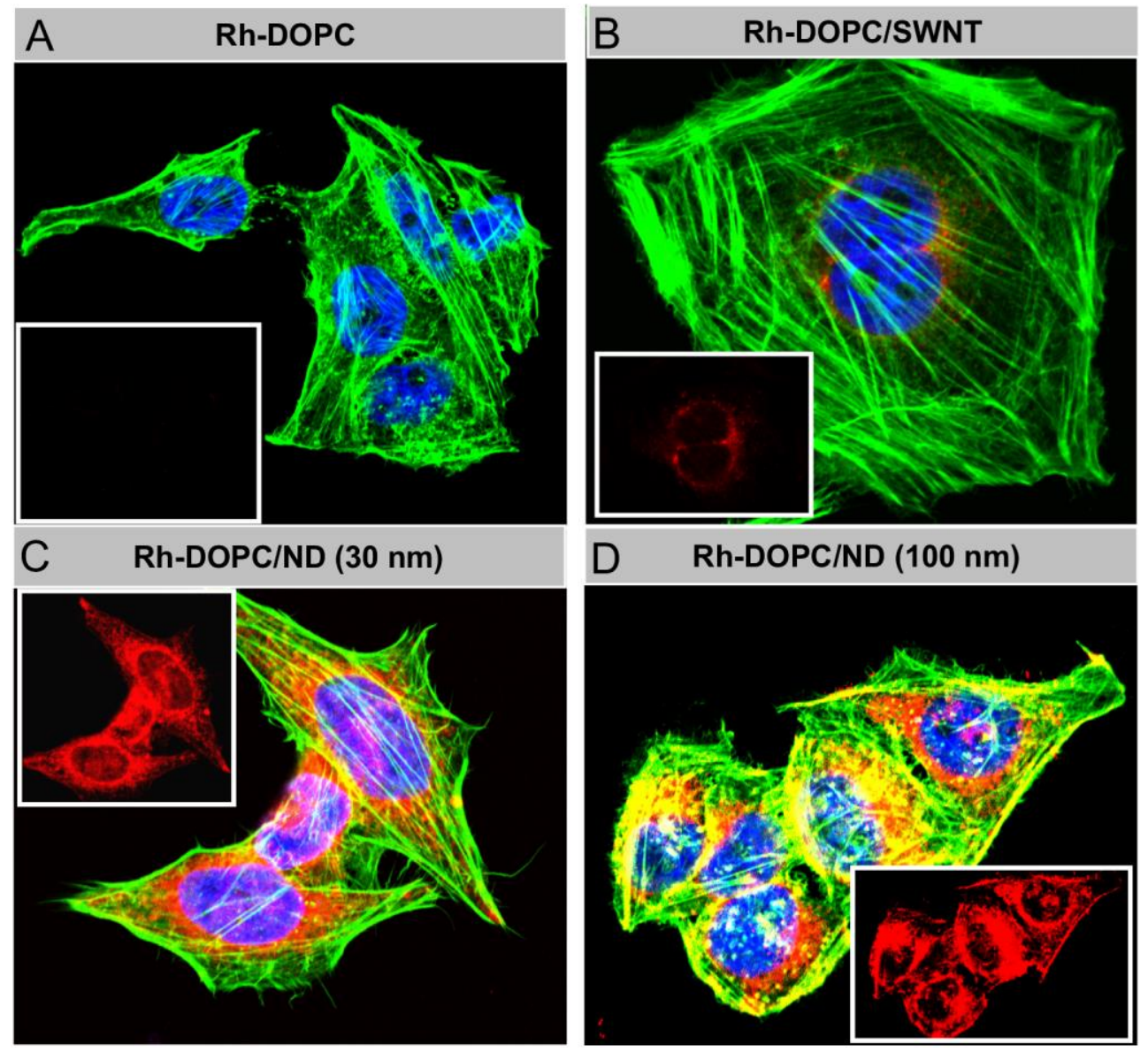

Figure 4. (A) Confocal fluorescence micrographs of HeLa cells incubated with free Rh-DOPC liposomes (A), Rh-DOPC/CNT (B), Rh-DOPC/30 nm ND (C) and Rh-DOPC/100 nm ND (D) for 4 hr. The cytoskeleton actin was stained in green and the nucleus was stained in blue. Inset: the red channel showing liposome fluorescence. 
After establishing the feasibility of using DOPC/ND as a delivery vehicle, we next tested its performance. Doxorubicin (DOX) is a commonly used anti-cancer drug and we compared its adsorption capacity on the nano-carbons. NGO has the highest capacity followed by CNT (Figure S11). This is understandable since all the carbon atoms are on the surface for NGO and CNT, while NGO can adsorb DOX on both sides. Each $\mathrm{mg}$ of ND can adsorb $\sim 0.2 \mathrm{mg}$ of DOX and the presence of DOPC does not change the capacity. To test multiplexed delivery, we employed calcein-loaded DOPC liposomes. After adding ND and incubating the complex with DOX, the vehicle was incubated with the HeLa cells (Figure 5A). Strong calcein (green) and DOX (red) fluorescence were observed, indicating co-delivery of both. For comparison, calcein-loaded free DOPC liposomes were not internalized by the cells (Figure 5B).
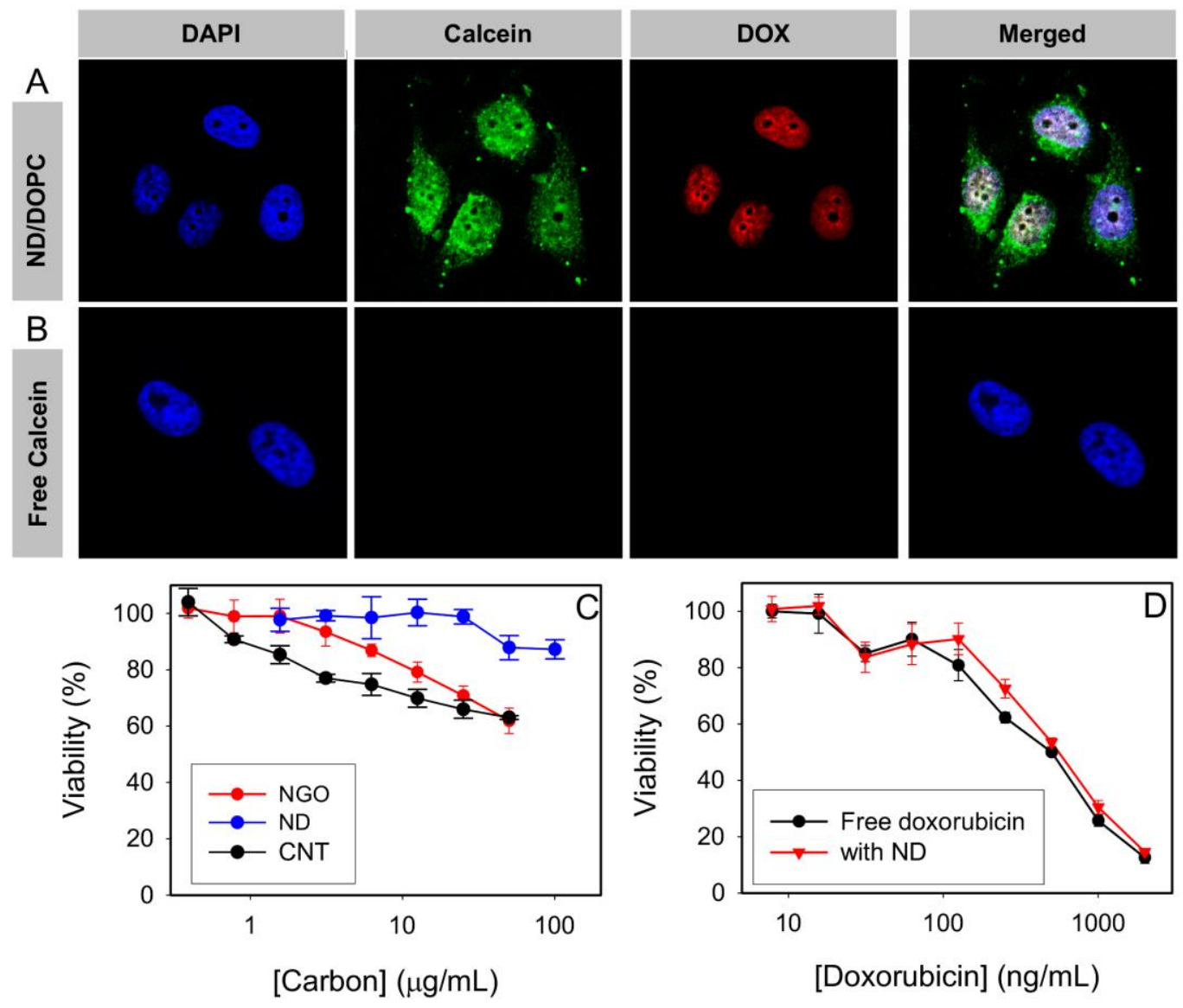
Figure 5. (A) Confocal fluorescence micrographs of co-delivery of calcein and DOX using DOPC/ND. (B) Delivery of calcein loaded DOPC liposomes alone. Cell viability measured using MTT assay for various nano-carbons (C) and the DOPC/ND complex with DOX or free DOX (D).

Next, we compared the toxicity of the nano-carbons. Consistent with the literature, ND has very low toxicity (Figure 5C); no growth inhibition was observed at below $20 \mu \mathrm{g} / \mathrm{mL}$ ND. ${ }^{43-45}$ Higher toxicity was observed with the two $s p^{2}$ carbons. When DOX was loaded, similar cancer cell killing efficacy was observed for the DOPC/ND vehicle compared to the free DOX. This suggests that most of the DOX added to the vehicle was delivered into the cells and released inside the cells. Since DOX can efficiently diffuse through the cell membrane, it is commonly to observe that a delivery vehicle does not enhance its efficacy. ${ }^{46,47}$ Using a drug carrier on the other hand allows potential targeted delivery to cancer cells while free DOX can also kill healthy cells. This study presents an interesting example of the interaction of different nano-carbons with liposomes and the cellular uptake of the complexes. Both the surface chemistry of carbon and geometry appear to be important.

2.5. Effect of lipid composition. Most of the above work was based on DOPC liposomes complexed with ND or other nano-carbons. Since the property of a liposome can be further tuned by introducing other lipid compositions, to achieve a complete understanding, we also studied the effect of adding charged lipids, fusogenic lipids (e.g. 1,2-dioleoyl-sn-glycero-3-phosphoethanolamine (DOPE)) and cholesterol. The main component was still DOPC to ensure adsorption and good biocompatibility. For all these studies, the imaging parameters were adjusted to be the same so that the amount of liposome uptake can be directly compared. First, as shown in the upper row of Figure 6, very little free liposomes were internalized, except for the sample with 10\% DOTAP (Figure 6C). This is consistent with the notion that DOTAP is cationic and attracted by the negatively charged cell membrane. Increase of liposome uptake by adding ND was observed for all the samples, and the sample containing $30 \%$ DOPE showed the highest level of uptake (Figure 6E). Therefore, using ND to assist liposome uptake 
is a general property of this system. DOPE is also a zwitterionic lipid, where its relatively small head group renders its fusogenic property. Introducing cholesterol appears to be detrimental for the liposome uptake (Figure 6D, F), while just 10\% negatively charged DOPG inhibits the uptake (Figure 6B). We confirmed this to be related to the reduced ND adsorption by the negatively charged liposomes (Figure 6G, Figure S12). Therefore, optimization of the cellular uptake property can be further achieved by varying the lipid composition.

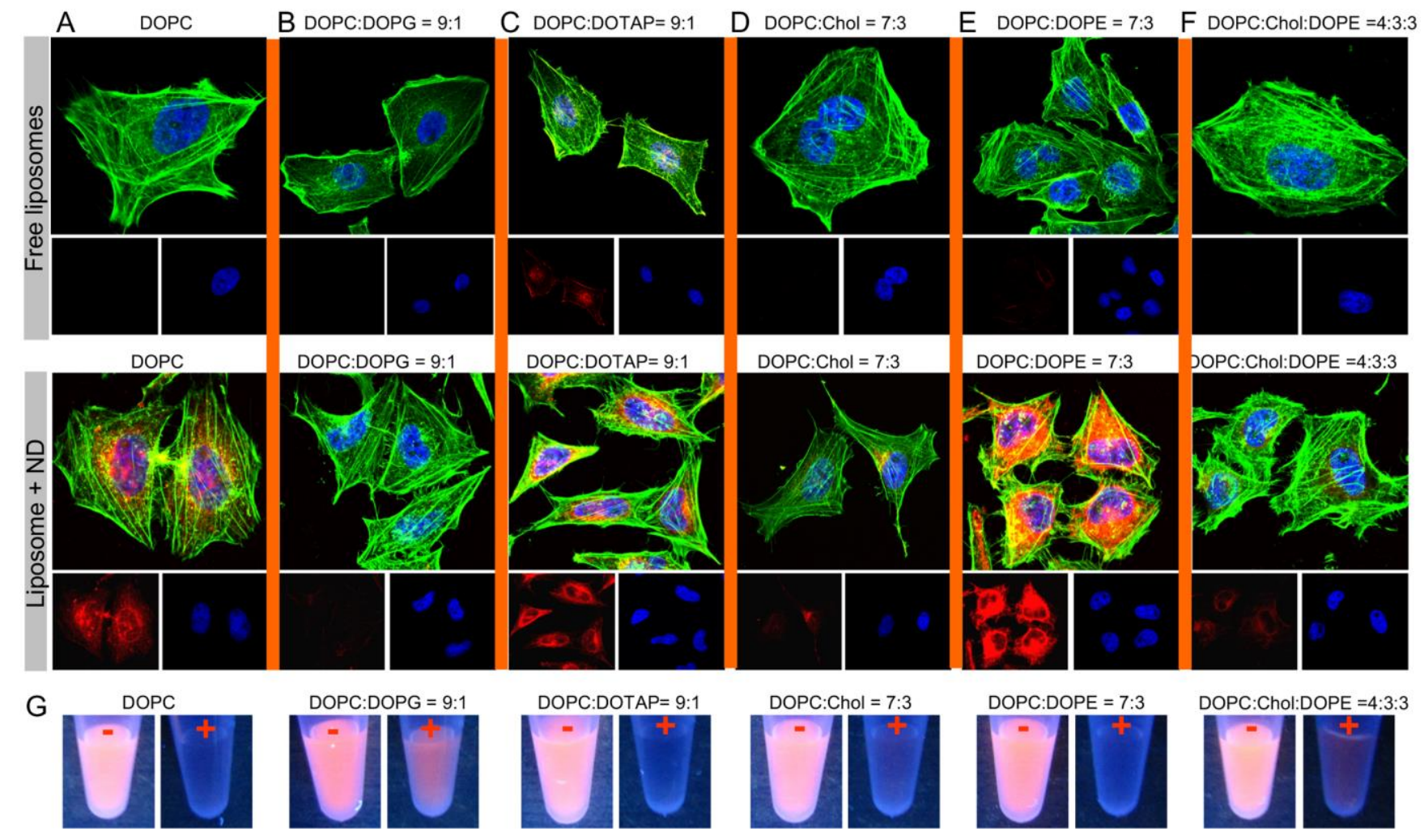

Figure 6. (A-F) Confocal fluorescence microscopy of HeLa cells incubated with free Rh-labeled liposomes (upper row) or with ND/liposome complex (bottom row) with various lipid compositions. Each sample is split into a red channel (Rh-lipid), a blue channel (cell nuclei) and a merged channel also with green fluorescence for the cell actin. (G) Photographs of free Rh-labeled liposomes (the tubes 
on the left) and the supernatant when the same liposome was mixed with ND (the tubes on the right). Chol $=$ cholesterol .

\section{MATERIALS AND METHODS}

Chemicals. All the phospholipids were purchased from Avanti Polar Lipids (Alabaster, AL). Monocrystalline nanodiamond powders (MSY 0-0.03, 0-0.05, 0-0.1) were purchased from Microdiamant AG (Lengwil, Switzerland). Carboxyl-modified single-walled carbon nanotubes (CNT) were purchased from SkySpring Nanomaterials Inc. (Houston, TX). GO was purchased from ACS Material, LLC (Medford, MA). Disodium calcein, doxorubicin hydrochloride, Triton X-100 and 3-(4,5dimethylthiazol-2-yl)-2,5-diphenyltetrazolium bromide (MTT) were obtained from Sigma-Aldrich (St Louis, MO). Dimethyl sulfoxide (DMSO), chloroform, 4-(2-hydroxyethyl)-1-piperazineethanesulfonic acid (HEPES) and $\mathrm{NaCl}$ were purchased from Mandel Scientific (Guelph, ON, Canada). LysoTracker Green, 4',6-diamidino-2-phenylindole (DAPI), 6-dodecanoyl-2-dimethylaminonaphthalene (Laurdan), and Alexa Fluor 488 phalloidin were from Life Technologies. All the cell culture related chemicals including medium, sera, and antibiotics were purchased from Fisher Scientific Inc. Mill-Q water was used to prepare all the buffers and solution. All other reagents and solvents were of analytical grade and used as received.

Preparation of ND. Monocrystalline ND powder with sizes of 30, 50 and $100 \mathrm{~nm}$ were carboxylated following the standard procedures. ${ }^{39}$ In brief, the ND powder sample $(0.5 \mathrm{~g})$ was heated in a 9:1 (v/v) mixture of concentrated $\mathrm{H}_{2} \mathrm{SO}_{4}$ and $\mathrm{HNO}_{3}(10 \mathrm{~mL})$ at $75{ }^{\circ} \mathrm{C}$ for 3 days. After centrifugation to remove the acid and washing in water, the samples were soaked in $\mathrm{NaOH}(0.1 \mathrm{M})$ at $90{ }^{\circ} \mathrm{C}$ for $2 \mathrm{~h}$, and then in $\mathrm{HCl}(0.1 \mathrm{M})$ at $90^{\circ} \mathrm{C}$ for $2 \mathrm{~h}$. The resulting carboxyl NDs were extensively rinsed with deionized water and Milli-Q water and harvested using ultracentrifugation at 50,000 rpm and dried. 
Preparation of NGO. The large GO sheets were dispersed in water at a concentration of $0.5 \mathrm{mg} \mathrm{mL}^{-1}$ and sonicated on an ultrasonic processor at a power of 120 watt $20 \mathrm{kHz}$ with pulse on for $2 \mathrm{~s}$ and pulse off for $4 \mathrm{~s}$ for $10 \mathrm{~h}^{12}$ After the treatment, the size of the GO sheets was decreased from micrometer to below $100 \mathrm{~nm}$.

Liposome preparation. Liposomes were prepared using the standard extrusion method. ${ }^{48}$ The miniextruder was purchased from Avanti Polar Lipids. DOPC, DOPG or DOPC/DOTAP (1:1, w/w) with a total mass of $2.5 \mathrm{mg}$ were respectively dissolved in chloroform. Rh-labeled liposomes were prepared by mixing $1 \% \mathrm{Rh}-\mathrm{PE}$ with various lipids in chloroform. Chloroform was then removed under a gentle $\mathrm{N}_{2}$ flow in the fume hood and trace amounts of residual chloroform was removed by storing the samples in a vacuum oven overnight at room temperature. The dried lipids were kept under a $\mathrm{N}_{2}$ environment and then stored at $-20{ }^{\circ} \mathrm{C}$ prior to use. To prepare liposomes, the lipids were hydrated with $0.5 \mathrm{~mL}$ buffer A (100 mM NaCl, $20 \mathrm{mM}$ HEPES, $\mathrm{pH}$ 7.6) at room temperature with occasional sonication for at least $2 \mathrm{~h}$. Therefore, the lipid concentration was $5 \mathrm{mg} \mathrm{mL}^{-1}$. The resulting cloudy suspension was extruded through two stacked polycarbonate membrane (pore size $=100 \mathrm{~nm}$ ) for 21 times. After extrusion, the lipid solution appeared to be transparent, indicating downsize of the lipid structures and formation of liposomes.

Calcein-loaded liposome preparation. To encapsulate calcein, the above prepared DOPC lipid film was hydrated with $100 \mathrm{mM}$ disodium calcein solution overnight with occasional sonication to help dispersing the lipid. ${ }^{49}$ The sample was then extruded using $100 \mathrm{~nm}$ pore sized membrane and free calcein was removed by passing the samples through a Pd-10 column using buffer A for elution, where the first $600 \mu \mathrm{L}$ of the fluorescent fraction was collected. The calcein concentration in the fraction is estimated to be $\sim 45 \mu \mathrm{M}$ based on the fluorescence intensity after rupturing the liposomes with Triton $\mathrm{X}$-100. To prepare calcein loaded DPPC liposomes, the hydration step using the $100 \mathrm{mM}$ calcein solution was carried out at $55^{\circ} \mathrm{C}$ for $2 \mathrm{hr}$ with occasional sonication to assist mixing. All the 
subsequent extrusion operations were carried out at this temperature. After extrusion, the free calcein was removed using the Pd-10 column and this step was carried out at room temperature as described above.

Laurdan-loaded liposome preparation. Laurdan loaded DOPC liposomes were prepared by mixing $10 \mu \mathrm{L}$ Laurdan $(1 \mathrm{mM})$ with $500 \mu \mathrm{L}$ DOPC lipid $\left(5 \mathrm{mg} \mathrm{mL}^{-1}\right)$ in chloroform (Laurdan : DOPC lipid molecule $=1: 318$ ). Then similar procedures were carried out for extrusion as mentioned above. Note that the Laurdan dye was loaded in the hydrophobic bilayer region. Laurdan loaded DPPC liposomes were prepared in a similar way but all the operations including hydration and extrusion were carried out at $55^{\circ} \mathrm{C}$.

Adsorption of liposome by nano-carbons. To understand the adsorption of the various charged liposomes to ND, the concentration of ND was fixed at $1 \mathrm{mg} \mathrm{mL}^{-1}$ in buffer (10 mM HEPES, $\mathrm{pH}$ 7.6). To $100 \mu \mathrm{L}$ of the ND solution, $1 \mu \mathrm{L}$ Rh-labeled zwitterionic DOPC liposomes, anionic DOPG liposomes and cationic DOTAP liposomes $\left(5 \mathrm{mg} \mathrm{mL}^{-1}\right)$ were added, respectively. To study the adsorption of various nano-carbons by DOPC liposomes, $1 \mu \mathrm{L}$ Rh-DOPC liposomes $\left(5 \mathrm{mg} \mathrm{mL}^{-1}\right) \mathrm{were}^{-}$ added to $100 \mu \mathrm{L} \mathrm{ND}\left(1 \mathrm{mg} \mathrm{mL}^{-1}\right)$, CNT $\left(50 \mu \mathrm{gL}^{-1}\right)$ and NGO $\left(50 \mu \mathrm{g} \mathrm{mL}^{-1}\right)$ in buffer $(10 \mathrm{mM} \mathrm{MES}$, $\mathrm{pH}$ 6.0), respectively. After centrifugation at $70,000 \mathrm{rpm}$ to precipitate the nano-carbons, the fluorescence of the samples was observed under UV light in a dark room. The supernatant fluorescence was photographed using a digital camera. The same procedures were used to observe liposome adsorption by ND in different $\mathrm{pH} . \mathrm{pH}$ was adjusted with a final of $10 \mathrm{mM}$ citrate buffer $(\mathrm{pH} 3,4,5,6)$ or HEPES buffer ( $\mathrm{pH}$ 7.6). $\mathrm{NaOH}$ was used to adjust $\mathrm{pH}$ to 9 and 11. To further confirm hydrogen bonding, DOPC liposomes were incubated with the three nano-carbons at pH 6 with 0, 2, 4, and $6 \mathrm{M}$ urea. The fluorescence was observed under a handheld UV lamp excitation in a dark room. For documentation, a digital camera was used (Canon PowerShot SD 1200 IS). 


\section{CONCLUSIONS}

In summary, we reported a simple method to attach ND to zwitterionic liposomes based on hydrogen bonding. The ND/liposome complex is highly stable and is disrupted only with $4 \mathrm{M}$ urea or at high $\mathrm{pH}$. For comparison, CNTs are adsorbed much weaker. Both DOPC liposomes and ND are highly biocompatible, making this design attractive for drug delivery. Another advantage is that both the interior of liposome and the ND surface can be used for drug loading. We demonstrated this by codelivery of calcein and doxorubicin. The adsorption of nano-carbons to the liposomes has very little effect on the membrane integrity or $T_{\mathfrak{c}}$, suggesting that the nano-carbons are only adsorbed by the membrane surface without disrupting the packing of the hydrophobic lipid tails. Therefore, the toxicity of these nano-carbons might not be related to their direct interaction with the cell membrane. Further optimization for cellular uptake has been achieved by introducing fusogenic DOPE lipid. Overall, this work has opened a door to rational engineering of the lipid/nano-carbon interface based on weak intermolecular forces.

\section{SUPPORTING INFORMATION}

Additional methods, IR, adsorption kinetics, TEM, confocal micrographs, DOX adsorption capacity. This material is available free of charge via the Internet at http://pubs.acs.org.

\section{ACKNOWLEDGMENT}

Funding for this work is from the University of Waterloo, the Canadian Foundation for Innovation, the NSERC of Canada and the Early Researcher Award from the Ontario Ministry of Research and Innovation.

\section{REFERENCES}

1. N. W. S. Kam, M. O'Connell, J. A. Wisdom and H. J. Dai, Proc. Natl. Acad. Sci. U.S.A., 2005, 102, 11600-11605. 
2. G. S. Hong, J. C. Lee, J. T. Robinson, U. Raaz, L. M. Xie, N. F. Huang, J. P. Cooke and H. J. Dai, Nat. Med., 2012, 18, 1841-1846.

3. H. Jin, D. A. Heller, R. Sharma and M. S. Strano, ACS Nano, 2009, 3, 149-158.

4. Y. R. Wu, J. A. Phillips, H. P. Liu, R. H. Yang and W. H. Tan, ACS Nano, 2008, 2, 2023-2028.

5. Z. Liu, J. T. Robinson, X. M. Sun and H. J. Dai, J. Am. Chem. Soc., 2008, 130, 10876-10877.

6. $\quad$ K. Yang, L. Feng, X. Shi and Z. Liu, Chem. Soc. Rev., 2013, 42, 530-547.

7. X. M. Sun, Z. Liu, K. Welsher, J. T. Robinson, A. Goodwin, S. Zaric and H. J. Dai, Nano Res., 2008, 1, 203-212.

8. K. Yang, S. A. Zhang, G. X. Zhang, X. M. Sun, S. T. Lee and Z. A. Liu, Nano Lett., 2010, 10, 3318-3323.

9. Y. Wang, Z. H. Li, D. H. Hu, C. T. Lin, J. H. Li and Y. H. Lin, J. Am. Chem. Soc., 2010, 132, 9274-9276.

10. L. Feng and Z. Liu, Nanomedicine, 2011, 6, 317-324.

11. H. Wang, Q. Zhang, X. Chu, T. Chen, J. Ge and R. Yu, Angew. Chem. Int. Ed., 2011, 50, 70657069.

12. F. Wang, B. Liu, A. C. F. Ip and J. Liu, Adv. Mater., 2013, 25, 4087-4092.

13. C. S. Wang, J. Y. Li, C. Amatore, Y. Chen, H. Jiang and X. M. Wang, Angew. Chem. Int. Ed., $2011, \mathbf{5 0}, 11644-11648$.

14. V. N. Mochalin, O. Shenderova, D. Ho and Y. Gogotsi, Nat Nano, 2012, 7, 11-23.

15. A. M. Schrand, S. A. C. Hens and O. A. Shenderova, Critical Reviews in Solid State and Materials Sciences, 2009, 34, 18-74.

16. Y. Zhu, J. Li, W. X. Li, Y. Zhang, X. F. Yang, N. Chen, Y. H. Sun, Y. Zhao, C. H. Fan and Q. Huang, Theranostics, 2012, 2, 302-312.

17. A. Krueger, Chem. Eur. J., 2008, 14, 1382-1390.

18. H. Huang, E. Pierstorff, E. Osawa and D. Ho, Nano Lett., 2007, 7, 3305-3314. 
19. Y.-C. Lin, E. Perevedentseva, L.-W. Tsai, K.-T. Wu and C.-L. Cheng, J. Biophotonics, 2012, 5, 838-847.

20. D. A. Ho, ACS Nano, 2009, 3, 3825-3829.

21. V. N. Mochalin and Y. Gogotsi, J. Am. Chem. Soc., 2009, 131, 4594-+.

22. C. C. Fu, H. Y. Lee, K. Chen, T. S. Lim, H. Y. Wu, P. K. Lin, P. K. Wei, P. H. Tsao, H. C. Chang and W. Fann, Proc. Natl. Acad. Sci. U.S.A., 2007, 104, 727-732.

23. Y. Xing and L. M. Dai, Nanomedicine, 2009, 4, 207-218.

24. A. E. Nel, L. Madler, D. Velegol, T. Xia, E. M. V. Hoek, P. Somasundaran, F. Klaessig, V. Castranova and M. Thompson, Nat. Mater., 2009, 8, 543-557.

25. W. Jiang, B. Y. S. Kim, J. T. Rutka and W. C. W. Chan, Nat. Nanotechnol., 2008, 3, 145-150.

26. D. Peer, J. M. Karp, S. Hong, O. C. FaroKhzad, R. Margalit and R. Langer, Nat. Nanotechnol., 2007, 2, 751-760.

27. M. Bally, K. Bailey, K. Sugihara, D. Grieshaber, J. Voros and B. Stadler, Small, 2010, 6, 24812497.

28. V. P. Torchilin, Nat. Rev. Drug Discov., 2005, 4, 145-160.

29. A. J. L. Villaraza, A. Bumb and M. W. Brechbiel, Chem. Rev., 2010, 110, 2921-2959.

30. R. Frost, G. E. Jonsson, D. Chakarov, S. Svedhem and B. Kasemo, Nano Lett., 2012, 12, 33563362.

31. P. K. Ang, M. Jaiswal, C. H. Y. X. Lim, Y. Wang, J. Sankaran, A. Li, C. T. Lim, T. Wohland, O. Barbaros and K. P. Loh, ACS Nano, 2010, 4, 7387-7394.

32. A. V. Titov, P. Kral and R. Pearson, ACS Nano, 2009, 4, 229-234.

33. A. C. F. Ip, B. Liu, P.-J. J. Huang and J. Liu, Small, 2013, 9, 1030-1035.

34. F. Karchemski, D. Zucker, Y. Barenholz and O. Regev, J. Control. Release, 2012, 160, 339-345.

35. E. Miyako, K. Kono, E. Yuba, C. Hosokawa, H. Nagai and Y. Hagihara, Nat Commun, 2012, 3, 1226. 
36. X. Zhou, J. M. Moran-Mirabal, H. G. Craighead and P. L. McEuen, Nat Nano, 2007, 2, 185-190.

37. L. Moore, E. K.-H. Chow, E. Osawa, J. M. Bishop and D. Ho, Adv. Mater., 2013, 10.1002/adma.201300343.

38. A. Bumb, S. K. Sarkar, N. Billington, M. W. Brechbiel and K. C. Neuman, J. Am. Chem. Soc., 2013.

39. P. H. Chung, E. Perevedentseva, J. S. Tu, C. C. Chang and C. L. Cheng, Diam. Relat. Mater., $2006,15,622-625$.

40. T. Parasassi, G. De Stasio, G. Ravagnan, R. M. Rusch and E. Gratton, Biophys. J., 1991, 60, 179-189.

41. A. F. Xie, R. Yamada, A. A. Gewirth and S. Granick, Phys. Rev. Lett., 2002, 89, 246103.

42. B. Wang, L. F. Zhang, S. C. Bae and S. Granick, Proc. Natl. Acad. Sci. U.S.A., 2008, 105, 18171-18175.

43. H. F. Wang, S. T. Yang, A. N. Cao and Y. F. Liu, Acc. Chem. Res., 2013, 46, 750-760.

44. X. Zhang, W. Hu, J. Li, L. Tao and Y. Wei, Toxicol. Res., 2012, 1, 62-68.

45. N. Mohan, C.-S. Chen, H.-H. Hsieh, Y.-C. Wu and H.-C. Chang, Nano Lett., 2010, 10, 36923699.

46. S. P. Sherlock, S. M. Tabakman, L. Xie and H. Dai, ACS Nano, 2011, 5, 1505-1512.

47. L. Zhang, J. Xia, Q. Zhao, L. Liu and Z. Zhang, Small, 2010, 6, 537-544.

48. N. Dave and J. Liu, ACS Nano, 2011, 5, 1304-1312.

49. N. Dave and J. Liu, Adv. Mater., 2011, 23, 3182-3186. 